\title{
ITEM RESPONSE MODELS \\ FOR THE MEASUREMENT OF THRESHOLDS
}

\author{
Richard D. Morey
}

Jeffrey N. Rouder, Dissertation Supervisor

\begin{abstract}
At least since Fechner (1860) described examples of human sensory thresholds, the concept of a threshold has been foundational in psychology. Thresholds exist when a sensation can be so weak that it does not lead to detection. Recently, however, thresholds have been abandoned in psychology as a result of the advent of the Theory of Signal Detection (Green \& Swets, 1966). I argue that this abandonment was premature and that the concept of a threshold is useful in psychological theory. Thresholds may be defined as the maximum stimulus intensity for which performance is equal to a chance baseline. The measurement of thresholds, however, remains a difficult problem. I present statistical models designed to allow the efficient measurement of thresholds. The models, which have much in common with Item Response Theory models, are hierarchical and are analyzed by Bayesian methods. The models perform well both in simulation and in application to data. Finally, I apply the general model to data from two subliminal priming experiments to test the phenomenon of subliminal priming.
\end{abstract}

